\title{
Bell's Spaceships Problem and the Foundations of Special Relativity
}

Francisco Fernflores

Recent 'dynamical' approaches to relativity by Harvey Brown and his colleagues have used John Bell's own solution to a problem in relativity which has in the past sometimes been called 'Bell's spaceships paradox', in a central way. This paper examines solutions to this problem in greater detail and from a broader philosophical perspective than Brown et al. offer. It also analyses the well-known analogy between special relativity and classical thermodynamics. This analysis leads to the sceptical conclusion that Bell's solution yields neither new philosophical insights concerning the foundations of relativity nor differential support for a specific view concerning the existence of space-time.

\section{Introduction}

Recently, Brown (2005) and Brown and Pooley $(2001,2006)$ have used a problem in special relativity concerning a pair of co-accelerating spaceships and a string, originally posed by Dewan and Beran (1959) and later restated by Bell (1993), hereafter 'the DBB problem', as part of a rather subtle argument for their 'dynamical' interpretation of relativity, which includes the contention that Minkowski space-time is not a real entity. The weight of neither Brown and Pooley's dynamical approach to relativity nor their anti-realist conclusion rests exclusively on their discussion of Bell's own solution to the DBB problem. However, Brown does cite Bell's solution as a paradigm of how we ought to understand the familiar relativistic effect of length contraction using the dynamical approach to relativity (Brown 2005).

The purpose of this article is to examine the DBB problem and its solutions in a broader philosophical context that is independent of arguments concerning the reality of space-time. The primary goal here is to explore the consequences of the DBB problem, if there are any, both for our understanding of length contraction and, 
more broadly, for the philosophical foundations of special relativity. Additionally, we will pursue in some detail the famous analogy between classical thermodynamics and special relativity, which Einstein framed explicitly as early as 1919 (Einstein 1982, 228), for this is an analogy that both Brown and Bell use. We shall argue that once solutions to the DBB problem are properly understood and the analogy between special relativity and classical thermodynamics is clarified, the sceptical conclusion that the DBB problem offers no new insights concerning either the foundations of special relativity or the ontology of the theory is difficult to resist. Because our motivations and philosophical methods are substantially different from those of Brown and his colleagues, we do not engage in a point-by-point criticism of their views but indicate only philosophically significant differences where necessary.

The article begins, in Section 2, with a statement of the DBB problem. In Section 3, we contrast the standard static case of length contraction, which we illustrate with Pauli's discussion of Einstein's 1911 twin-rod thought experiment, with the dynamic case of length contraction in the DBB problem. Even in the latter, however, one can choose to analyse the physical system in question from a purely kinematical point of view, which we explain in Section 4. We also fill a lacuna in such kinematical solutions to the DBB problem by introducing the notion of momentary proper length, with which one can tell a consistent story concerning how the proper length of the string changes in the DBB problem. In Section 5, we distinguish dynamical solutions to the DBB problem into phenomenological solutions and solutions from first principles. We discuss Cornwell's phenomenological solution in Section 6 and Bell's solution from first principles in Section 7, where we emphasize features of Bell's solution not noted by Brown and his colleagues. In Section 8, we provide a detailed examination of the analogy between special relativity and thermodynamics and use it to illuminate Bell's own use of this analogy and seemingly conflicting claims in the literature concerning the nature of length contraction. We conclude, in Section 9, that if these philosophical lessons are correct, it becomes more difficult to see Bell's solution as offering support for either any specific interpretation of special relativity or a specific philosophical view about the reality of space-time.

\section{The DBB Problem}

When Bell (1993) reintroduced a problem originally discussed by Dewan and Beran (1959) to illustrate his non-standard pedagogical approach to relativity, he did so roughly as follows. Consider three identical spaceships $A, B$, and $C$ arranged in a line in two-dimensional Minkowski space-time in a state of relative rest in an inertial frame $F$ with $C$ equidistant between $A$ and $B$. A light, taught string is stretched between equally placed protrusions on $A$ and $B$ (Dewan 1963), though one routinely idealizes the spaceships as point particles. $C$ emits a light pulse in opposite directions toward $A$ and $B$. When $A$ and $B$ receive the light signal, they accelerate executing identical acceleration programmes' (Bell 1993, 67) as they travel along the positive $x$-direction of the Cartesian coordinate system $S$ adapted to $F$. Does the string eventually break? 
The answer to this question has, in the past, eluded even experts, as Bell famously reports (Bell 1993, 68), though it would not do so today. A growing number of contributions to the physics literature, which routinely assume for pragmatic reasons, as we will, that the ships accelerate with identical constant proper accelerations, show unequivocally that if the string is light enough and the ships accelerate for long enough, the string will indeed break - see, for example Dewan and Beran (1959), Bell (1993), Cornwell (2005), Flores (2005), the recent exchange between Redžić and Peregoudov (Redžić 2008; Peregoudov 2009; Redžić 2009), and references therein. Furthermore, some of these contributions show that the standard geometrical approach to special relativity is sufficient to arrive at the conclusion that the string will indeed eventually break. Nevertheless, understanding how these solutions can be categorized as either kinematical or dynamical and understanding their respective scope, especially when we focus not on whether the string breaks but on how the length of the string changes, can yield a better understanding of the analogy between classical thermodynamics and special relativity. However, before explaining these two types of solution, let us note how the DBB problem differs from the standard cases of length contraction discussed in elementary textbook presentations of relativity (e.g. French 1968; Rindler 1991; Taylor and Wheeler 1992) and qualitative presentations of relativity by philosophers (e.g. Maudlin 1994; Kosso 1998; Lange 2002).

\section{Standard Cases of Length Contraction and the DBB Problem}

In the standard static case of length contraction, one compares the length of an extended object $W$ originally at rest in an inertial frame $F$-which remains at rest in $F$ as the proper time $\tau_{W}$ elapses, as measured by a clock on, say, one of the spatial end points of $W$-with the length of $W$ as reckoned in a different inertial frame $F^{\prime}$, where $F^{\prime}$ moves with a constant non-zero velocity $v$ relative to $F$. This is the type of length contraction Einstein first discussed in 1905 (Einstein 1952a), Minkowski interpreted geometrically in 1908 (Minkowski 1952), and Pauli described as 'not a property of a single measuring rod taken by itself, but ... a reciprocal relation between two such rods moving relatively to each other' (Pauli 1958, 12-13). Pauli describes length contraction as a relationship between rods because he is assuming, as Einstein originally did, that the length of a rod is a primitive notion in relativity that one uses to define distance. Significantly, Pauli reaches his characterization of length contraction through an examination of Einstein's 1911 twin-rod thought experiment, which merits our attention.

Consider two rods $A_{1} B_{1}$ and $A_{2} B_{2}$ of equal rest length $l_{0}$ whose spatial end points are $A_{1}, B_{1}$ and $A_{2}, B_{2}$, respectively. Let $S$ be an inertial coordinate system in which the two rods move with equal and opposite velocity $v$. Because of the symmetry of the configuration, the end points of the rods $A_{1}$ and $A_{2}$, and $B_{1}$ and $B_{2}$, will overlap simultaneously in $S$. Finally, suppose that when $A_{1}$ coincides with $A_{2}$ and $B_{1}$ coincides with $B_{2}$, we mark on another rod at rest relative to $S$ these spatial end points and designate the marks $A^{*}$ and $B^{*}$, respectively. According to Pauli, the distance $l$ between $A^{*}$ and $B^{*}$ will satisfy $l=l_{o} / \gamma(v)$, where $\gamma(v)$ is the usual Lorentz factor. 
Einstein's twin-rod thought experiment compares the length of one of the two 'moving' rods, say $A_{1} B_{1}$, in its rest frame $F$ relative to which it continues to remain at rest with the length of that same object $A_{1} B_{1}$ relative to another inertial frame $F^{\prime}$ that moves with a velocity $v$ relative to $F$. From this perspective, the role of the second $\operatorname{rod} A_{2} B_{2}$ and the marking procedure that generates $A^{*} B^{*}$ is merely to avoid using clocks to ensure that one is reckoning the length of $A_{1} B_{1}$ along a simultaneity slice for $F^{\prime}$. Consequently, Pauli is correct that this thought experiment shows that (a) the rest length of $A_{1} B_{1}$ does not change but (b) there is a reciprocal relation of length contraction among objects at rest in $F$ and objects at rest in $F^{\prime}$.

In contrast to this standard static case of length contraction, the DBB problem involves comparing the length of an object $W$ originally at rest relative to the inertial frame $F$, relative to which the object then accelerates in a very specific way as proper time $\tau_{\mathrm{W}}$ elapses, with the length of $W$ relative to a different inertial frame $F^{\prime}$ relative to which $W$ is at rest at some later proper time $\tau_{\mathrm{W}}$. Strictly speaking, in the configuration of the DBB problem, this comparison can be conducted only after all parts of $W$ have ceased to accelerate and $W$ has come to rest in $F^{\prime}$. During the acceleration period, there does not exist an inertial frame relative to which all parts of $W$ are at rest, and hence the notion of a proper length for $W$ is not defined.

In the standard static case, nothing happens to $W$ despite the reciprocal relation of length contraction, because $W$ is in a state of uniform motion. There is no dynamics in this static case, in the straightforward sense that we are considering a physical configuration in which no forces (or fields) affect the motion of $W$. Additionally, $W$ has no internal dynamics; or at least one can say that whatever internal physical processes occur, $W$ remains in a state of equilibrium and none of its macroscopic, observable quantities change. Whence, the standard static case of length contraction is often called a 'kinematical effect'.

However, in the DBB problem, the physical system of interest has a non-trivial dynamics. There are external forces acting on the spatial end points of the string $W$ as the engines accelerate the ships. These external forces are transmitted to the interior of $W$ where, as internal forces, they 'pull' the parts of $W$ along. Consequently, $W$ does not remain in a state of internal equilibrium and whatever happens to $W$ is not merely a 'kinematical effect', since it is the result of a combination of non-trivial external and internal dynamics. This last qualification is important, for there is one clear and wellknown case where $A$ and $B$ accelerate, i.e. where the spaceships-string system has an external dynamics, but where there is no interesting internal dynamics.

Consider a case where $A$ and $B$ do not execute 'identical acceleration programmes' but instead accelerate in such a way that at any instant, as reckoned by an inertial observer momentarily co-moving with either $A$ or $B$, any part of the string $W$ is at rest relative to any other part of the string. In this case, $W$ executes Born rigid motion (BRM), as described by, for example, Pauli (1958, 131-132) and Rindler $(1991,34)$. An object that executes BRM moves in such a way that, as judged by an inertial observer momentarily co-moving with either one of its spatial end-points, its proper length remains constant. Thus, if the spaceships had executed BRM, then the string would experience no relativistic stresses and if the ships ran out of fuel 
after the appropriate amount of proper time elapsed for each of them so that $A$ and $B$ would begin to coast simultaneously in the same inertial frame $F^{\prime}$, the final length of the string would be equal to its original length. Thus, in this case, although the external dynamics is non-trivial, the accelerations on each part of the string are selected precisely so as not to give rise to any internal changes to $W$, i.e. so as not to disturb $W$ out of its state of internal equilibrium. Precisely what physical process can produce such accelerations is, of course, a subtle, and a different, question altogether. Nevertheless, since the internal state of $W$ does not change as $W$ executes BRM, one can classify this as a case of static length contraction.

\section{Kinematical Solutions to the DBB Problem}

Although the spaceships-string system in the DBB problem has a non-trivial dynamics, one can choose to analyse its motion from a purely kinematical perspective, i.e. by neglecting the sources of acceleration and the details of how the forces are transmitted within the system. Of course, in such a treatment one recognizes that one is bracketing important details of the relevant physics. Nevertheless, such a kinematical analysis is useful both because it yields the qualitative solution that if the string is light enough and the ships accelerate for long enough, the string will break, and because with additional quantitative stipulations, it yields quantitative results concerning when and where the string will break-see, for example, Rindler's problem 13 (Rindler 1991, 37).

One obtains the qualitative solution by focusing on the initial and final states of the ships-string system. Suppose the two ships $A$ and $B$ in the DBB problem are originally a distance $d$ apart in $S$. Furthermore, suppose that to avoid using the notion of the length of a rod as a primitive in our reasoning we reckon distances using the familiar radar signalling method. One can define the distance $D_{A B}$ from $A$ to $B$ as reckoned by $A$ at an event $a \in A$, where we now use $A$ and $B$ also to designate the world lines of the ships, where no ambiguity threatens. The distance $D_{A B}$ is the amount of time, as measured by a momentarily co-moving inertial observer (MCIO) $I$ to $A$ at $a$, that light takes to travel from $A$ to $B$, which $I$ can measure in light seconds, say, by bouncing a light pulse off $B$ and dividing the time of flight of the light pulse in half. Prior to the acceleration period, $D_{A B}=d$ and clearly $D_{B A}=D_{A B}$, where $D_{B A}$ is the obvious analogue of $D_{A B}$. Once all acceleration has ceased, an elementary calculation shows that $D_{A B}=d \cdot \gamma(v)=D_{B A}$, where $\gamma(v)$ is the familiar Lorentz factor and $v$ is the final velocity of the ships relative to $F$. So, for example, if the final speed of the ships in $S$ is one half the speed of light, the distance $D_{A B}=d \cdot \gamma(v)$ the string must be able to span, once all acceleration has ceased, is approximately $1.15 d$. If the string is not flexible enough to stretch this far, or to any lesser value in the interval $(d, d \cdot \gamma(v)]$, then the string must break.

One can thus solve the DBB problem in at least two equally satisfying ways. One can say that although the coordinate distance between $A$ and $B$ in $S$ is constant and equal to $d$ throughout the entire history of the ships (as described in $F$ ), the distance the string must span in its final rest frame after all acceleration has ceased is not $d$ but rather the 
longer distance $d \cdot \gamma(v)$. Alternatively, one can reason counterfactually. In order for the string to remain taught at the same tension, without experiencing any additional stretching forces, the ships $A$ and $B$ would have to execute BRM. Had the ships been executing BRM, the coordinate distance from $A$ to $B$ in $S$ would diminish and, after all acceleration had ceased, it would have been less than $d .{ }^{1}$ However, since the final coordinate distance in $S$ between the two ships is equal to $d$, the string was stretched. One can append to this kinematical account an explanation of how for $A$, $B$ is pulling ahead since $A$ measures the distance $D_{A B}$ to $B$ along $A$ 's increasingly inclined simultaneity slices whereas for analogous reasons, $B$ reckons that $A$ is falling farther and farther behind-see, for example, Flores (2005).

The kinematical solution to the DBB problem leaves the following question unanswered: how exactly does the proper length of the string change during the acceleration period? Somewhat surprisingly, there is no straightforward answer to this question in a kinematical analysis of the DBB problem, because once the ships begin accelerating, there does not exist an inertial frame relative to which the front and back ends of the string are in a state of relative rest. There is no such thing as 'the rest frame of the string' once acceleration begins until after all acceleration has ceased and the string comes to rest in $F^{\prime}$, assuming it does not break, of course. Thus, we can only say that before the ships began accelerating the proper length of the string was $d$ and, assuming either that the ships did not accelerate for long enough to break the string or that the string was flexible enough to withstand the stretching, the proper length of the string after all acceleration ceased is $d \cdot \gamma(v)$. However, it seems we must remain silent about precisely how the proper length of the string changes during the acceleration period. Is this the best we can do?

An analysis of the conditions for the applicability of the notion of proper length seems to indicate otherwise. Suppose one asks: what are the necessary conditions for the applicability of the notion of proper length? One can state these conditions in 'coordinate-free' language as follows:

1. There exists a space-like line $\Sigma$ that bisects $W$ so that at the points of intersection $a$ $\in A$ of $\Sigma$ and $A$ and $b \in B$ of $\Sigma$ and $B$, the four-velocities $\mathbf{v}_{\boldsymbol{a}}$ of $A$ and $\mathbf{v}_{\boldsymbol{b}}$ of $B$ are parallel (using the well-defined notion of parallel four-vectors and appealing to the obvious two-dimensional versions of the four-dimensional vectors).

2. The four-velocity $\mathbf{v}_{\boldsymbol{a}}$-and necessarily $\mathbf{v}_{\boldsymbol{b}}$ because of (1) above-is orthogonal to the space-like line $\Sigma$.

In the two-dimensional cases we are considering, it is a straightforward exercise to show that (1) and (2) obtain both in the familiar case of an object $W$ that moves inertially in Minkowski space-time and in the case of an object whose parts execute BRM.

There are two telling cases where the conjunction of conditions (1) and (2) is not true. First, consider an object $W$ whose spatial end points $A$ and $B$ move inertially with a non-zero constant relative velocity $v$. There does not exist a space-like line $\Sigma$ such that the four-velocities $\mathbf{v}_{\boldsymbol{a}}$ and $\mathbf{v}_{\boldsymbol{b}}$ are parallel. Thus, condition (1) is false and the notion of proper length does not apply to $W$. There does exist an inertial frame 
in which the magnitudes of the relevant velocities are equal, but in that frame the velocities have opposite directions. Intuitively, $W$ does not have a proper length, because there does not exist an inertial frame in which its spatial end points are simultaneously at rest. Note that even in this case one can define the distance $D_{A B}$ between $A$ and $B$ at an event $a \in A$ and its analogue $D_{B A}$. Thus, this case is particularly useful for distinguishing the notion of distance from the notion of length: a proper length is always a distance between the spatial end-points of an object but the distance between the spatial end-points of an object is not always a proper length.

The second case to consider is the accelerating portions of $A$ and $B$ in the DBB problem. The notion of proper length does not apply to the string while either one of the ships is accelerating, because the conjunction of conditions (1) and (2) above is false. However, it is only condition (2) that is false, for condition (1) is true in this case. This is significant for it entails that there exists a pair of world lines, which we can construct by extending $\mathbf{v}_{\boldsymbol{a}}$ and $\mathbf{v}_{\boldsymbol{b}}$, that can be regarded as the world lines $U_{1}$ and $U_{2}$ of the spatial end points of an inertially moving object $U$ to which the notion of proper length does apply. This object $U$ is the two-dimensional analogue of an MCIO as $U$ momentarily co-moves with the string $W$. Figure 1 displays the relevant spacetime diagram for $U$ and $W$, which momentarily co-move when $U_{1}$ is coincident with $A$ at event $a$ and $U_{2}$ is coincident with $B$ at event $b .{ }^{2}$ The events $a$ and $b$ are simultaneous in F. Figure 2 displays the relative spatial location of $U$, the spaceships, and the string $W$ at a series of equally separated coordinate times (in $S$ ). $U$ and $W$ both move left to right. $U$ moves with constant coordinate velocity $v=v_{a}$ in $S$, while the ships move with increasing coordinate velocity $w$. At coordinate time $t_{a}$, $W$ moves with coordinate velocity $w=v$, and $U_{1}$ and $U_{2}$ momentarily co-move with $A$ and $B$ respectively.

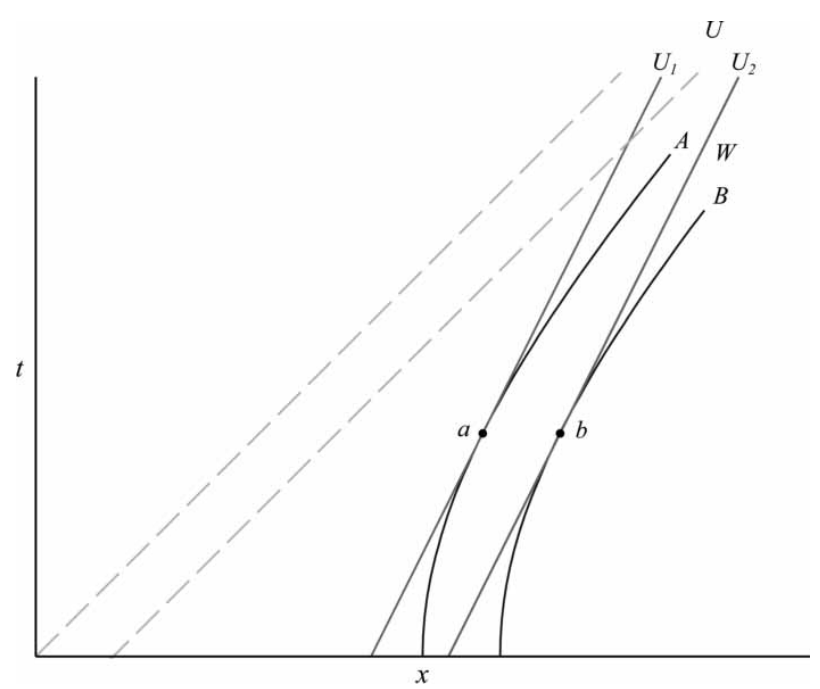

Figure 1 Space-time diagram for accelerating phase of Bell's spaceships problem. The inertially moving object $U$ and the string $W$ momentarily co-move when $U_{1}$ is coincident with $A$ at event $a$ and $U_{2}$ is coincident with $B$ at event $b$. 

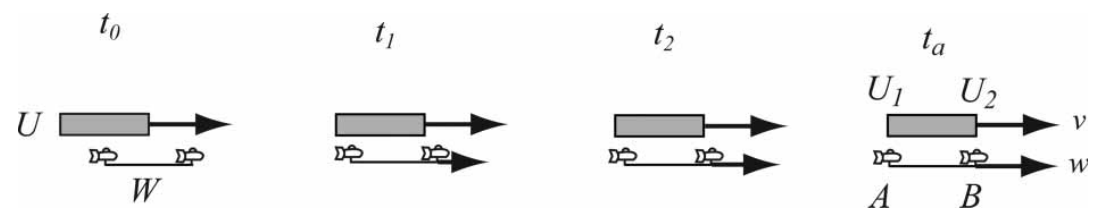

Figure 2 Space diagram illustrating the relative positions of the inertially moving object $U$, the spaceships, and the string $W$ at a series of equally separated coordinate times in the original rest frame $S$ of the ships-string system.

The observation that the ships $A$ and $B$ momentarily co-move with $U_{1}$ and $U_{2}$, respectively, at coordinate time $t_{a}$ suggests that we can define the momentary proper length $\lambda$ of the string $W$ at the 'instant' picked out by the events $a$ and $b$, i.e. when the proper times for $A$ and $B$ respectively satisfy $\tau_{a}=\tau_{b}$, as the value of the proper length of $U^{3}$ Notice that so defined, during the acceleration phase, $\lambda$ is neither equal to the coordinate distance $d$ (in $S$ ) between the spaceships nor to the coordinate length of $U$ (in $S$ ). The value of $\lambda$ is also not equal to either one of the distances $\left.D_{A B}\right|_{a}$ or $\left.D_{B A}\right|_{b}$. Yet, one might ask, why should we regard $\lambda$ as having anything to do with the length of the string $W$ ?

First, notice that as stated the question is ill-posed, for it presupposes that there is such a thing as 'the length of the string'. However, the term 'the length of the string' is too ambiguous to designate any specific quantity. If we interpret 'the length of the string' as meaning 'the proper length of the string', we are closer to asking a meaningful question. Still, the question may be entirely inappropriate, precisely because there is no such thing as 'the proper length of the string' once acceleration has begun. A better way to ask our question is: can we define a quantity that can reasonably be interpreted as the proper length of the string 'at an instant', where we also specify how such an 'instant' is to be selected (as the notion of 'at an instant' is obviously not Lorentz invariant)? There are two considerations that suggest that our $\lambda$ is such a quantity.

First, consider the situation from the ' $3+1$ ' view depicted in Figure 2. Suppose that the ships and the string $W$ cease to accelerate when $U_{1}$ is coincident with $A$ at the event $a$ and $U_{2}$ is coincident with $B$ at the event $b$, i.e. when $A$ and $B$ have each reached the coordinate velocity $w=v$. Furthermore, suppose that in $S$ the coordinate length $l$ of $U$ is equal to the coordinate length $d$ of $W$ when $w=v$. After acceleration has ceased, we can conclude that the proper length $\lambda$ of both $U$ and $W$ is $\lambda=d \cdot \gamma\left(v_{a}\right)$. Notice that the 'instant' is picked out by the coincidence of the world lines $U_{1}$ and $A$ at the event $a$ and $U_{2}$ and $B$ at $b$.

Yet, that $W$ stopped accelerating should not matter. Consider the following analogue to Einstein's twin-rod thought experiment. Suppose when $U_{1}$ is coincident with $A$ and $U_{2}$ is coincident with $B$, two marks $M_{1}$ and $M_{2}$ are made on a rod at rest in $F$. The coordinate distance (in $S$ ) between these two marks is clearly $d$. The momentary proper length $\lambda$ of $U$ 'when' the marks were made, i.e. at the instant picked out by the two events ' $U_{1}$ is coincident with $A$ ' and ' $U_{2}$ is coincident with $B$ ', satisfies $\lambda=d \cdot \gamma\left(v_{a}\right)$. Finally, since at that 'instant' not only were the spatial end points of $W$ coincident with the spatial end points of $U$ but the end points of both $U$ and $W$ moved with 
the same coordinate velocity and hence were in a state of relative rest, we can say that $\lambda$ is the momentary proper length of $W$. Of course, none of this reasoning depends on the specific values of the proper times $\tau_{a}$ and $\tau_{b}$ for the events $a \in A$ and $b \in B$, respectively, so long as $a$ and $b$ occur in the accelerating portion of $A$ and $B$ and $\tau_{a}$ $=\tau_{b}$. Consequently, one can say that at the proper time $\tau_{a}=\tau_{b}$, where $a$ and $b$ are any such simultaneous events in $F$, the momentary proper length of the string $W$ is $\lambda$.

Second, suppose we 'linearize' the curved trajectories of $A$ and $B$ so that they become the concatenation of a series of 'small' line segments, as depicted in Figure 3. We do this by concatenating first-order approximations of $A$ and $B$ at a series of events on $A$ and $B$, respectively, separated by equal intervals of proper time. Each corresponding pair of line segments that approximates the curves $A$ and $B$ can be regarded as the spatial end points of an inertially moving object $W_{i}$ that co-moves with another inertial object $U$ of proper length $\lambda$ for a small and finite amount of proper time. Thus, while the string 'occupies' the rest frame of $U$, its proper length is $\lambda$. So long as the line segments are long enough, it also happens that $D_{A B}=D_{B A}$ and $D_{A B}=\lambda$. However, as we consider the limit in which the line segments become infinitesimally small, the quantities $D_{A B}, D_{B A}$, and $\lambda$ are no longer equal. ${ }^{4}$

One can easily verify that so defined, the value of the momentary proper length $\lambda$ for an arbitrary object $W$ is always equal to the proper length of $W$ both when $W$ moves inertially and when $W$ s parts execute BRM. Furthermore, it is clear that $\lambda$, which is a Lorentz-invariant quantity, reduces to the corresponding Newtonian notion in the appropriate limit, though defining such a notion in the Newtonian context is otiose.

Still, while one might agree that $\lambda$ is mathematically well-defined and has the properties just enumerated, one could argue that it is the interpretation of $\lambda$ as the momentary proper length that is questionable since we seem to have arbitrarily privileged an inertial frame (maybe $F$ or the rest frame of $U$ ). However, this is not the case. As we have suggested above, the 'moment' at which $\lambda$ is defined is picked out by the Lorentzinvariant criterion (1) for the applicability of the notion of proper length. Because we

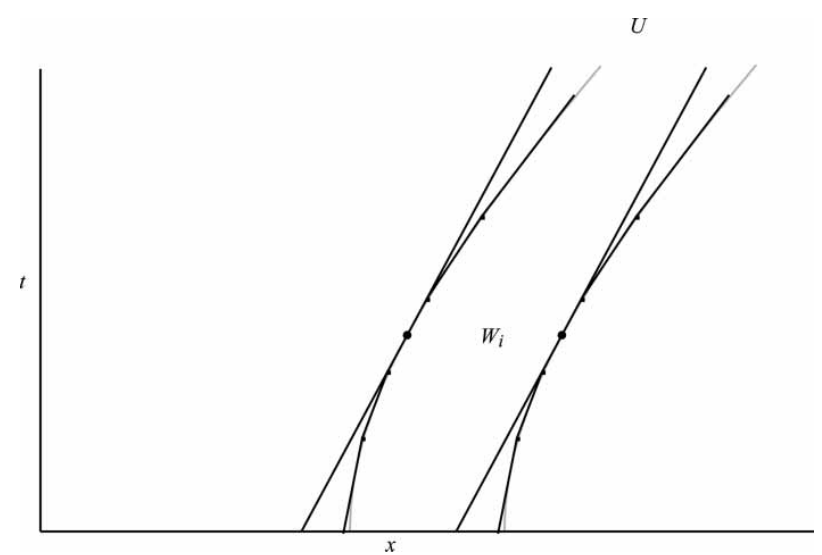

Figure 3 A first-order approximation of the curved trajectories $A$ and $B$, illustrating that the string momentarily 'occupies' the rest frame of $U$ with which it is coincident for a small but finite amount of proper time. 
are assuming that the two ships in the DBB problem move with the same constant proper acceleration, which is a sufficient but not necessary condition for the ships to be executing 'identical acceleration programmes', for any given event $a$ in the accelerating portion of $A$, there exists a unique event $b \in B$ such that the four-velocities $\mathbf{v}_{\boldsymbol{a}}$ and $\mathbf{v}_{\boldsymbol{b}}$ are parallel. This entails that the momentarily co-moving object $U$ we construct is also unique and hence the same for all inertial observers.

Consequently, one can use the momentary proper length $\lambda$ to give a consistent account concerning the proper length of the string in the DBB problem. We no longer have to say that we must remain entirely silent about the proper length of the string while it accelerates, only to discover that once all acceleration has ceased, the proper length of the string has increased. Instead, one can say that prior to the events at which $A$ and $B$ begin accelerating, the value of $\lambda$ for the string is constant and equal to $d$. The momentary proper length $\lambda$ then increases monotonically, since $\lambda=d \cdot \gamma\left(v_{m}\right)$, where $v_{m}$ is the velocity of $A$ relative to $S$ at an event $m$ on the accelerating portion $A$. If the string withstands the stresses that occur during the accelerating phase, then its final momentary proper length is just $\lambda=d \cdot \gamma\left(v_{f}\right)$, where $v_{f}$ is the final velocity of $A$ and $B$ in $S$. Finally, note that, at the very least, condition (1) for the applicability of proper length is also a necessary condition for the applicability of the momentary proper length. Thus, it does not follow that there always exists a 'moment' at which we can apply the notion of momentary proper length for an object whose spatial end points move arbitrarily.

\section{Types of Dynamical Solutions to the DBB Problem}

There are two types of dynamical solutions to the DBB problem: phenomenological solutions and solutions a physicist might describe as solutions 'from first principles'. Bell's solution to the DBB problem (Bell 1993) is of the latter type. The former type of solution, which Cornwell (2005) offers, has been generally overlooked by philosophers. Before examining philosophically Cornwell's and Bell's solutions, the following brief remarks on the distinction between these two types of dynamical solutions seem germane.

In general, the contrast between a phenomenological solution to a problem and a solution from first principles has an entrenched use among physicists. Roughly, it is used to indicate the difference between solving a problem by attending to quantities that are comparatively easily measured and typically characterize macroscopic features of a physical system, with solutions that attend to quantities that are comparatively difficult to measure and typically characterize microscopic features of a physical system. This contrast is most famously exemplified in the difference between classical, phenomenological thermodynamics and the kinetic theory of gases. However, it is also exemplified in the difference, say, between calculating the current in a conductor phenomenologically, e.g., using Ohm's law, versus calculating the same current from first principles as one does by providing a theoretical description of the microscopic constituents of the conductor in solid-state physics. It may even be, though this is somewhat speculative, that this is the type of distinction Einstein tried to convey to the 
public in his newspaper article for the London Times in 1919 when he described the difference between 'principle' and 'constructive' theories (Einstein 1982, 228). Thus, this classification of dynamical solutions to the DBB problem is not intended to introduce yet another philosophical distinction between 'levels' of theories but is intended merely to describe the physics in a way that is amenable to both physicists and philosophers.

\section{Cornwell's Phenomenological, Dynamical Solution}

The string in the $\mathrm{DBB}$ problem experiences differential forces once the acceleration period begins. A phenomenological approach to the DBB problem attends to the relevant forces, masses, and accelerations and aims to derive at least the conditions under which the string must break. Additionally, one can use this approach to calculate, as Cornwell does, for a given string, the amount of time one would have to wait in order for the string to break, assuming, of course, that the ships do not run out of fuel first and begin to coast. However, as Cornwell suggests, because one is attending to the dynamics of the problem, the problem itself needs to be more carefully specified. As formulated by Bell, the DBB problem treats the dynamical effects of the string on the ships-string system as negligible and hence ignorable. However, in more realistic versions of the problem, the string, or 'cord' as Cornwell calls it, exerts a force on the ships and affects their state of motion. For these reasons, Cornwell restates the DBB problem as follows:

Two identical ships are floating in flat space at relative rest, the one directly in front of the other, and with a cord tied between them, attached at identical points. At the same moment, both ships gradually fire up their identical engines to an identical steady thrust, doing so identically according to identical clocks located at identical places in the ships. They run their engines for long enough to reach half light speed relative to the original rest frame. What happens to the cord? Does it break or does it not? (Cornwell 2005, 699)

Immediately, Cornwell explains that the correct answer to the question is 'it depends'. If the string is strong enough, the entire ships-string system will execute something very close to BRM and the string will not break. If the string is not strong enough, the trajectories of the ships deviate appreciably from the trajectories of ships executing BRM, and the string will break. Notice that in this scenario, although the two ships fire their engines 'identically', in the sense Cornwell specifies in the above quotation, their world lines are not 'parallel' in $S$, i.e. $A$ cannot be brought into coincidence with $B$ by performing a spatial translation unless we ignore the dynamical effects of the string on the ships. A closer examination of Bell's requirement that the two ships execute 'identical acceleration programmes' illustrates the main difference in Cornwell's formulation of the problem.

Bell nowhere states precisely what he means by stipulating that the ships in the DBB problem execute 'identical acceleration programmes'. However, a reasonable interpretation of this requirement can be developed as follows. Let us assume, with Bell, that $A$ and $B$ are identical ships. Let us further assume that they carry identical clocks (with which to measure proper time $\tau$ ) and identical accelerometers (with which to measure 
proper acceleration). Finally, let us assume that when $A$ and $B$ receive the light signal from $C$, they set their clocks to $\tau=0$. One can then define:

The two spaceships $A$ and $B$ 'have' or 'execute' identical acceleration programmes if, and only if, their respective accelerometers record equal values of proper acceleration for equal values of elapsed proper time.

More abstractly, an acceleration program is simply a set of ordered pairs where the first element is a value of proper time and the second element is a value for the proper acceleration of the ship. Physically, 'identical acceleration programmes' can be realized, for example, by having identical on-board computers with identical acceleration programs adjust the throttle on the ships' engines continuously so that each ship achieves the desired value of proper acceleration at the desired proper time (cf. Cornwell 2005, 702). Notice that, on this interpretation, ships that co-accelerate with the same constant proper acceleration instantiate only one special case of 'identical acceleration programmes'.

One can easily construct an acceleration program so that after the acceleration period ends, the length of the string is any value greater than or even equal to $d$. As an example of the latter, consider an acceleration program in which the two ships accelerate with the same constant proper acceleration for an amount of proper time $\tau_{0}$ and then decelerate with the same constant proper acceleration for the same amount of proper time $\tau_{0}$ and hence return to a state of rest relative to $S$ after all acceleration has ceased. Physically, this acceleration program could be realized by ships that have identical engines front and back and the appropriate navigation computers (as described above). The back engine of the ship fires only for an amount of proper time $\tau_{0}$ at which instant (assuming that we can ignore issues concerning the relativity of simultaneity that arise if we consider the ship to a have non-negligible spatial separation between the two engines), the identical front engine fires only for an amount of proper time $\tau_{0}$. If the string can withstand the stresses 'during' the acceleration phase (roughly speaking), it will not break and will return to its original length $d$ once all acceleration has ceased. Even a purely kinematical analysis of the problem yields this conclusion. Thus, the answer to Bell's own version of the DBB problem is actually the same as the answer to Cornwell's problem, i.e., 'it depends', though for importantly different reasons.

For Cornwell, the reason why the answer to his problem is 'it depends' is that he is including the non-trivial dynamics of the string in the DBB problem. Cornwell suggests that we approach the problem by considering the force that the string would have to exert in order for the two ships to execute BRM. If at some time the string cannot exert this force on the ships, because it is not strong enough, then the string must break. Cornwell estimates that, if we assume that the original length of the string is $L$, the acceleration of the mid-point of the string is $A$ and the ships have a mass $M$, the entire ships-string system executes rigid motion if the string is able to exert a force $F \approx(1 / 2)\left(M A^{2} L\right)$, in $c=1$ units. Cornwell then uses this approximation to develop the following example:

Consider a spider weighing one gram hanging from a thread it has spun which is of length one meter. Attach this between ships each weighing about one hundred 
metric tonnes. Ignoring the non-relativistic effects, how long would one have to wait to reach half the speed of light without the thread breaking? $T$ is easily calculated to be a little more than twenty minutes. (Cornwell 2005, 701)

Thus, Cornwell's phenomenological approach gives concrete results rather quickly. As is often the case, such results would be far more difficult to obtain from first principles.

\section{Bell's Dynamical Solution from First Principles}

Bell's approach to solving the DBB problem is quite different from Cornwell's; Bell wishes to show that the string contracts relative to the inertial frame $F$ because of the detailed physical processes that the constituents of the string experience. Bell assumes that the string is composed of Bohr-like atoms and sets out to determine, using Maxwell's theory, how the circular orbit of an electron will deform once its nucleus begins to accelerate gently. Bell admits, of course, that the model he adopts for the constituents of the string, governed as it is by an inadequate, non-quantum mechanical theory of matter, is rather crude. However, Bell's primary aim in his paper is pedagogical: he wishes to show that by using the theoretical resources with which students are already familiar, namely Maxwell's theory, one can derive some of the main results of relativity by working entirely within the context of one inertial frame. Furthermore, Bell quips, 'it is often simpler to work in a single frame, rather than to hurry after each moving object in turn' (Bell 1993, 77).

Determining changes to the shape of the circular electron orbit is, in outline, a straightforward calculation consisting of two main steps. First, one finds an expression for the field produced by the nucleus (ignoring the field produced by the electron itself) using Maxwell's equations. Second, one finds the electron's equation of motion for the electron in that field and solves it. However, the details of this calculation are rather subtle both because Bell must make several approximations, given the complexity of even this simple configuration, and because to derive length contraction Bell has to use what is in effect the relativistic definition of momentum, though he does not introduce it that way, for important reasons as we shall presently see.

The exact equations for describing the field produced by the gently accelerating nucleus of charge $Z e$, Bell explains (Bell 1993, 70), are known. These equations, which Bell provides in a footnote (Bell 1993, 78-79), are rather complex because even in classical electrodynamics-which is the theory Bell invokes-changes to the field some finite distance from its source do not happen instantaneously. Consequently, the exact field equations involve retarded potentials. Furthermore, there are good reasons for requiring the acceleration of the nucleus to be gentle, the most obvious of which is to ensure that the electron is not stripped from the nucleus. For these reasons, Bell suggests, we can instead use the equations produced by a charge $Z e$ that moves with a uniform velocity $V$, which, as Bell reports, 'differ only slightly' (Bell 1993, 70) from the exact field equations. Perhaps due to the nature of his article, Bell does not bother to state the accuracy of any approximation he makes in the paper, of which there are several including this one. He paints with rather broad brush strokes and leaves it to the industrious reader to fill in the details. 
The second step is to write down the equation of motion for the electron and solve it. To obtain the equation of motion for the electron, Bell uses the classical Lorentz force law, which following Bell, one can write as $\dot{\mathbf{p}}=-e(\mathbf{E}+\dot{\mathbf{r}} \times \mathbf{B})$, where $\mathbf{p}, \mathbf{E}$, and $\mathbf{B}$ are the familiar three-vectors for the momentum of the electron, the electric field, and the magnetic field, $\mathbf{r}$ is the position vector for the electron, and we are using cgs units with $c=1$. Ordinarily, one would rewrite this equation by using the Newtonian relationship between velocity, momentum, and mass $\dot{\mathbf{r}}=\mathrm{p} / m$ to eliminate $\dot{\mathbf{r}}$ in the Lorentz force law and obtain an equation of motion. However, Bell explains that this Newtonian relationship is inadequate because it implies that by acting long enough with a given electric field an electron could be taken to an arbitrarily high velocity' (Bell 1993, 70). Bell then explains that this is unacceptable because one finds empirically that the speed of light is a limiting velocity and that 'the experimental facts are fitted by a modified formula proposed by Lorentz' (Bell 1993, 70).

In special relativity, the formula Bell attributes to Lorentz is known simply as the definition of relativistic three-momentum, a formula which, for example, Einstein goes to great lengths to obtain in his 1935 derivation of mass-energy equivalence (Einstein 1935; Flores 1998) and which Mermin and Feigenbaum also deduce in their modified version of Einstein's original 1905 derivation of mass-energy equivalence (Mermin and Feigenbaum 1988). For Bell, however, the formula for the relativistic three-momentum, which one can write, following Bell, as $\dot{\mathbf{r}}=\mathbf{p} /\left(m^{2}+p^{2}\right)^{1 / 2}$, where $p^{2}$ is the squared (Euclidean) magnitude of $\mathbf{p}$, is obtained empirically. This implicitly imports another approximation into Bell's derivation, for such a formula is presumably obtained by carrying out measurements and then using a curve-fitting algorithm. More importantly, it is as if Bell wants to imagine how we might have arrived at relativistic results, such as length contraction, from a purely Newtonian understanding of the world supplemented only by an empirical finding that modifies the definition of momentum. If this is correct, then, it seems the dot over the $\mathbf{r}$ and $\mathbf{p}$ must indicate, though Bell nowhere says so, differentiation with respect to Newtonian time, and not with respect to either relativistic coordinate time or relativistic proper time.

To obtain the equation of motion, one then substitutes the empirically obtained expression for $\mathbf{r}$ and the expressions for the $\mathbf{E}$ and $\mathbf{B}$ fields produced by the nucleus Bell had previously obtained into the Lorentz force law to obtain a system of coupled differential equations that do not admit an exact solution. As Bell correctly notes, 'one can programme a computer to integrate these equations' (Bell 1993, 70). If one does, Bell continues, one finds that 'if the acceleration of the nucleus is sufficiently gradual, the initial circular orbit deforms slowly into an ellipse... the contraction is to a factor $\sqrt{1}-V^{2 / c^{2}}$, (Bell 1993, 70). Since the results of a numerical integration performed on a computer are numbers, we find here evidence of another approximation.

After making yet another approximation and deriving what Bell describes as the Lorentz transformations, though we can now appreciate that they are only approximately so, Bell suggests that one can easily expand the line of reasoning he has pursued to macroscopic objects. He says: 
by a trivial extension this reasoning applies not only to a single electron interacting with a single electromagnetic field, but to any number of charged particles, each interacting with the fields of all others. This allows an extension to very complicated systems of some of the results described above for the simple atom. (Bell 1993, 73)

Whether an extension of the reasoning is as trivial as Bell suggests and precisely which results derived for the simple atom can be so extended is rather subtle. As Bell himself points out, even if we focus only on one electron and the field produced by its nucleus, whether the acceleration in question is gentle enough depends on how 'tight' the orbit is. Consequently, the conclusion that the string will contract obtains only 'for a coherent dynamical system whose configuration is determined essentially by internal forces and only a little perturbed by gentle external forces accelerating the system' (Bell 1993, 75), which Bell is willing to assume. Bell then concludes:

In the rocket problem in the introduction, the material of the rockets, and of the thread, will Lorentz contract. A sufficiently strong thread would pull the rockets together and impose Fitzgerald contraction on the combined system. But if the rockets are too massive to be appreciably accelerated by the fragile thread, the latter has to break when the velocity becomes sufficiently great. (Bell 1993, 75)

One thus arrives, with Bell, at the qualitative conclusion obtained from both kinematical solutions and dynamical, phenomenological solutions. Furthermore, the above quotation indicates that Bell recognizes the value of dynamical, phenomenological solutions, for the last two sentences of the above quotation do not report results Bell has derived.

\section{The Analogy with Thermodynamics and Length Contraction}

An examination of the analogy between special relativity and classical thermodynamics can illuminate the relationship between the different solutions to the DBB problem and the philosophical foundations of special relativity. There are lessons to uncover not only concerning Bell's use of this analogy (Bell 1992), which Brown cites favourably (Brown 2005, 125-126), but also concerning seemingly conflicting claims about how to understand length contraction philosophically. For example, the title of Martínez's review of Brown's book (Brown 2005) rhetorically asks: 'There's no pain in the FitzGerald contraction, is there?' (Martínez 2007). Superficially, Martínez's question resembles Maudlin's when the latter tests his reader's comprehension of kinematical relativistic effects by asking 'What would it be like to travel at 99.99 percent the speed of light?' (Maudlin 1994, 57). While Maudlin's question challenges no one with even an elementary understanding of relativity, Martínez's question is more subtle especially if one appreciates that Bell is correct, in a sense, when he says that 'the Lorentz contraction can do physical damage' (Bell 1992, 34). The analogy between special relativity and classical thermodynamics helps one to resolve the apparent conflict in such claims.

As in ordinary classical mechanics, in thermodynamics one can distinguish static physical systems from dynamic ones. A simple example of the former is a gas in thermal equilibrium enclosed in a container that is completely isolated from its 
immediate surroundings. For such static gases one can use the ideal gas law to calculate any one of the familiar phenomenological state variables given the values of the other two. As an example of a dynamic physical system in thermodynamics, one only has to imagine that the gas container has a movable wall pushed by a piston and that the piston is moving relative to the walls of the container. In this case, because an external force is being applied (to move the piston) and is transmitted to the interior of the physical system, viz., the gas, the system has a non-trivial dynamics. Still, even in this case one can analyse the system by ignoring the dynamics and focusing on comparing final states. For example, one can use the ideal gas law when the piston is initially at rest, and then use it again after the piston has come to rest and enough time has elapsed so that the gas attains equilibrium, to determine how the state variables have changed through the compression process. One can carry this analysis even further if one ignores the internal dynamics of the gas, because one can use the ideal gas law to compare any two instantaneous states of the gas even when the piston is moving.

Arguably, the subtle relationship between the unsavouriness of leaving the ideal gas law as an unexplained brute fact and the promise of the molecular hypothesis yielding quantitative results motivates the familiar constructive explanation afforded by the kinetic theory of gases (KTG). More importantly, one can give an account of the observed relationships among the state variables in both the static and dynamic case. In a sense that is difficult to articulate and defend, KTG yields the greatest insight in dynamic cases, such as when one uses KTG to explain why as a piston compresses a gas the pressure on all sides of the container changes, which would not happen if one was compressing a solid object. An account of static cases using KTG merely constitutes a promising start. From a methodological perspective, if an adequate account of the static case cannot be found, there is little hope that a dynamic account is forthcoming.

Because the physical system in the DBB problem has non-trivial external and internal dynamics, it is most closely analogous to a dynamic system in thermodynamics, and not a static one. The example of the compression of a gas in a cylinder by a piston that begins and ends at rest provides a tolerably good analogue to the DBB problem. In both cases, the subsystem of interest, i.e. the gas in the one case and the string in the other, begins in a state of equilibrium, it is then disrupted from this state by an external force, until after the external force is no longer applied and enough time has elapsed the system returns to equilibrium, assuming the string does not break. As in thermodynamics, in the DBB problem one can opt — as kinematical solutions display-for a purely kinematical analysis of changes to the spaceships-string system that compares only final states and reaches important qualitative and quantitative conclusions. However, such an approach self-consciously ignores both the external and internal forces that act on the system to bring about the changes in question and assumes, for any pair of states it compares, that the system has either settled back into an equilibrium state or that its internal dynamics can be ignored 'at an instant', which is not an invariant notion in relativity and hence must be handled carefully. 
If this way of drawing the analogy between static and dynamic systems in both relativity and thermodynamics is broadly correct, one can use it to resolve the apparent conflict between the remarks made by Bell and Martínez about length contraction. When Bell compares thermodynamics and special relativity, he says:

If you are, for example, quite convinced of the second law of thermodynamics, of the increase of entropy, there are many things that you can get directly from the second law which are very difficult to get directly from a detailed study of the kinetic theory of gases, but you have no excuse for not looking at the kinetic theory of gases to see how the increase of entropy actually comes about. In the same way, although Einstein's theory of special relativity would lead you to expect the FitzGerald contraction, you are not excused from seeing how the detailed dynamics of the system also leads to the FitzGerald contraction. (Bell 1992, 34)

On a narrow interpretation of 'dynamics', in which one interprets examining 'the detailed dynamics of the system' as fruitful only for systems with non-trivial dynamics, Bell is encouraging us to look for a constructive account of length contraction only for dynamic cases of length contraction, such as the DBB problem. This is a reasonable interpretation of Bell's remarks because he focuses exclusively on the increase of entropy and does not mention idealized static, isentropic systems in thermodynamics analogous to static cases of length contraction.

On this interpretation, one is excused from analysing 'the detailed dynamics of the system' in static cases of length contraction, which are the types of cases on which Martínez (2007) focuses, because such systems have only a trivial dynamics. Martínez is correct that the Lorentz contraction does not hurt so long as we confine ourselves to static cases, which is no surprise. However, Bell is also correct, in a sense, because in dynamic cases, if the external dynamics of a system are not just right, i.e. if the forces are not applied in such a way that the system executes BRM, then physical damage can certainly result from external forces 'pulling' apart the string against its 'natural' tendency to contract, which is admittedly highly metaphorical language all solutions to the DBB problem make fairly precise. Yet, this cannot be the whole story.

The desire to complement the phenomenological account with an account from first principles is not unique to the analysis of physical systems with non-trivial dynamics, especially once a theory of the constituents of the system is forthcoming. As in the case of a static gas in thermodynamics, one should at least search for an account of the static case of length contraction based on a theory of matter $T$ from which one can derive the relationships among the state variables from first principles. The theory $T$ would have to be a Lorentz covariant quantum theory from which one can derive the length $l$ of an object in its own rest frame and the length $l^{\prime}$ of the same object as reckoned in a different inertial frame $F^{\prime}$. Significantly, even though Bell neglects the requirement that $T$ be a quantum theory, he does not begin by considering the static case first. Instead, he arrives at an approximation of the static case by first considering the dynamic case.

It is no accident that Bell's version of the DBB problem uses spaceships whose world lines $A$ and $B$ are both curved and parallel. The trajectories are curved because Bell anticipates the effects of accelerated motion on an idealized atom described by 
Maxwell's theory. The trajectories are parallel because Bell anticipates that in the lowacceleration limit, such trajectories reduce to inertial trajectories, which will enable him to derive, as he does, approximate versions of the Lorentz transformations. Furthermore, Bell simply assumes that the theory he uses to analyse the string can account for the string's length in the string's rest frame. However, this is not a trivial assumption for those interested in the foundations of relativity.

Bell nowhere discusses the meaning of the distance between the nucleus and the electron in the static case. Borrowing Einstein's 1916 language (Einstein 1952b, 115), one could say that Bell nowhere analyses the meaning of the spatial coordinates he uses to describe the motion of the electron. Yet, if Bell's solution to the DBB problem is supposed to provide a reductive explanation of length contraction analogous to reductive explanations afforded by KTG, he cannot employ Einstein's approach of analysing spatial distance in terms of the length of rigid rods that satisfy the laws of Euclidean geometry. Furthermore, Bell's approach cannot be improved upon by adopting the radar signalling method to define distance and then treating length as a derivative notion, which one can adopt in a kinematic and phenomenological solution to the DBB problem, as we have seen. The radar signalling method depends crucially on neglecting any momentum transfer that occurs as a light beam strikes an object and is reflected. Clearly, one cannot make this assumption at the subatomic level. Thus, if we regard the proper length $l$ of an object as a macroscopic state variable in Bell's solution to the DBB problem, it is analogous to volume in thermodynamics and not to either pressure or temperature, both of which are eliminated in the reduction to KTG. Thus, the likelihood that Bell's own solution to the DBB problem yields either new insights concerning the foundations of relativity or additional support to one side of the debate concerning the ontology of space-time seems significantly reduced.

\section{Conclusion}

Exploring the various ways in which the DBB problem can be solved illustrates that Bell's and Brown's preference for a constructive solution from first principles seems unlikely to support either an interpretation of relativity that emphasizes dynamics or a view about the unreality of Minkowski space-time. Instead, the analogy between special relativity and classical thermodynamics, if pursued a bit further than has hitherto been done, suggests that Bell's own solution to the DBB problem is analogous to the constructive explanation afforded by KTG of a dynamic physical system in thermodynamics. While such accounts certainly effect an eliminative reduction of some of the macroscopic state variables, other state variables, such as volume in thermodynamics and length in relativity, are not eliminated by such accounts. If this is correct, while a constructive solution to the DBB problem may help one understand the macroscopic dynamics of the string in terms of the dynamics of its constituents, and while one may even extend this approach to static cases of length contraction, it becomes more challenging to avoid the sceptical conclusion that the DBB problem sheds no new light on the foundations of special relativity. 


\section{Acknowledgements}

I would like to thank the two anonymous referees of this journal for their careful and constructive criticisms and Rachel Fernflores for her insightful comments on an earlier draft of this article.

\section{Notes}

[1] If the ships $A$ and $B$ execute BRM, then they do not execute 'identical acceleration programmes'. Instead, $A$ and $B$ move with very specific but different constant proper accelerations. Consequently the ships have to accelerate for different amounts of proper time before shutting off their engines to ensure the two ends of the string 'arrive' at the same inertial frame $F^{\prime}$.

[2] We created Figures 1 and 3 using Maple 13.

[3] This assumes, of course, that $A$ and $B$ set their clocks to read the same value of proper time, e.g. when they received the light signal from $C$ and began accelerating.

[4] The relationship between $D_{A B}, D_{B A}$, and $\lambda$ as a function of the elapsed proper time $\tau$ for either $A$ or $B$ is somewhat subtle. We explore this relationship and other relationships assumed in this paper analytically, and display the relevant plots, in Fernflores (forthcoming).

\section{References}

Bell, J. S. 1992. George Francis FitzGerald. Physics World 5: 31-35.

1993. How to teach special relativity. In J. S. Bell, Speakable and unspeakable in quantum mechanics, 67-80. Cambridge: Cambridge University Press.

Brown, H. R. 2005. Physical relativity: Space-time structure from a dynamical perspective. Oxford: Oxford University Press.

Brown, H. R., and O. Pooley. 2001. The origins of the spacetime metric: Bell's 'Lorentzian pedagogy' and its significance in general relativity. In Physics meets philosophy at the Planck scale, edited by C. Callender and N. Huggett, 256-272. Cambridge: Cambridge University Press.

- 2006. Minkowski space-time: A glorious non-entity. In The ontology of spacetime, edited by D. Dieks, 67-89. Oxford: Elsevier.

Cornwell, D. T. 2005. Forces due to contraction on a cord spanning between two ships. Europhysics Letters 71: 699-704.

Dewan, E. 1963. Stress effects due to Lorentz contraction. American Journal of Physics 31: 383-386.

Dewan, E., and M. Beran. 1959. Note on stress effects due to relativistic contraction. American Journal of Physics 27: 517-518.

Einstein, A. 1935. Elementary derivation of the equivalence of mass and energy. Bulletin of the American Mathematical Society 41: 223-230. - 1952a. On the electrodynamics of moving bodies. Reprinted in The principle of relativity, edited by H. A. Lorentz, H. Weyl, and H. Minkowski, 37-65. New York: Dover.

. 1952b. The foundations of the general theory of relativity. Reprinted in The principle of relativity, edited by H. A. Lorentz, H. Weyl, and H. Minkowski, 111-164. New York: Dover. . 1982. What is the theory of relativity? Reprinted in A. Einstein, Ideas and opinions, 227-232. New York: Crown.

Fernflores, F. Forthcoming. The kinematics of the Dewan-Beran-Bell spaceships problem.

Flores, F. 1998. Einstein's 1935 derivation of $E=m c^{2}$. Studies in History and Philosophy of Modern Physics 29: 223-243.

2005. Bell's spaceships: A useful relativistic 'paradox'. Physics Education 40: 500-503.

French, A. P. 1968. Special relativity. Cambridge, MA: MIT Press. 
Kosso, P. 1998. Appearance and reality: An introduction to the philosophy of physics. New York: Oxford University Press.

Lange, M. 2002. An introduction to the philosophy of physics: Locality, fields, energy, and mass. Malden, MA: Blackwell.

Martínez, A. A. 2007. There's no pain in the FitzGerald contraction, is there? Studies in History and Philosophy of Modern Physics 38: 209-215.

Maudlin, T. 1994. Quantum non-locality and relativity. Malden, MA: Blackwell.

Mermin, D. N., and M. J. Feigenbaum. 1988. $E=m c^{2}$. American Journal of Physics 56: 18-21.

Minkowski, H. 1952. Space and time. Reprinted in The principle of relativity, edited by H. A. Lorentz, H. Weyl, and H. Minkowski, 75-91. New York: Dover.

Møller, C. 1972. The theory of relativity. New York: Oxford University Press.

Pauli, W. 1958. Theory of relativity. New York: Dover.

Peregoudov, D. V. 2009. Comment on 'Note on Dewan-Beran-Bell's spaceship problem'. European Journal of Physics 30: L3-L5.

Redžić, D. V. 2008. Note on Dewan-Beran-Bell's spaceship problem. European Journal of Physics 29: N11-N19.

. 2009. Reply to 'Comment on "Note on Dewan-Beran-Bell's spaceship problem"'. European Journal of Physics 30: L7-L9.

Rindler, W. 1991. Introduction to special relativity. 2nd ed. New York: Oxford University Press.

Taylor, E. F., and J. A. Wheeler. 1992. Spacetime physics. 2nd ed. New York: W. H. Freeman. 